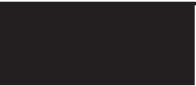

\title{
Simulating Trends in Reaction Path Geometry as a Function of External Fields. A Generalized Electronic Diabatic Model for Two-Dimensional Energy Surfaces
}

\author{
GUSTAVO A. ARTECA, ${ }^{1}$ JEAN PIERRE RANK, ${ }^{1}$ ORLANDO TAPIA ${ }^{2}$ \\ ${ }^{1}$ Département de Chimie et Biochimie and Biomolecular Sciences Programme, Laurentian University, \\ Ramsey Lake Road, Sudbury, ON, Canada P3E 2C6 \\ ${ }^{2}$ Department of Physical Chemistry, Uppsala University, Box 579, S-751 05 Uppsala, Sweden
}

Received 16 October 2007; accepted 24 January 2008

Published online 27 March 2008 in Wiley InterScience (www.interscience.wiley.com).

DOI 10.1002/qua.21645

\begin{abstract}
We introduce a protocol to represent quantum states as a linear superposition of model electronic diabatic basis states coupled in an external (static) electric field. By considering an entire family of these models, we uncover trends in reaction-path geometry and the topology of potential-energy surfaces, including all those that can be realized in a two-dimensional configurational space. Our approach can be used as a tool to model the key parameters (e.g., diabatic basis states, external field intensity) that yield desired geometrical characteristics in an actual potential energy surface. In this work, external agents such as laser fields, or a group of neighboring charges, are regarded as essential requirements to prompt, or trigger, the occurrence of a chemical process. In these cases, reaction path geometry can be modulated externally so as to yield processes that would appear to occur far from gas-phase geometries. This phenomenology is intrinsically nonadiabatic. Our present approach accounts for the possibility of such features, i.e., the occurrence of quantum states whose electronic structures resemble products, while at geometries that are more similar to those of reactants. @ 2008 Wiley Periodicals, Inc. Int J Quantum Chem 108: 1810-1820, 2008
\end{abstract}

Key words: diabatic states; potential energy surfaces; topology; structural similarity; reaction path geometry

Correspondence to: G. A. Arteca; e-mail: gustavo@laurentian.ca

Contract grant sponsor: NSERC, Canada; CRC, Canada. 


\section{Introduction}

$\mathbf{T}$ he stability of chemical species and the rate of chemical processes can be modulated by a number of external agents; typical examples include solvent effects, the field produced by an active site around a substrate or a catalyst's surface, as well as the laser control of some chemical reactions [1-12]. In cases, the environment changes only the energetics with little effect on reaction paths; this is typically found in many catalytic processes where the catalyst simply lowers effective energy barriers, or stabilizes intermediates, while maintaining the overall geometry of the path used to describe gasphase kinetics. In other situations, external factors can produce more remarkable modifications, giving rise to: (i) "off-path" geometries, e.g., stable intermediates with structures that are very different from those in gas-phase kinetics, and (ii) electronic species that resemble products, while at geometries that are more akin to reactants or intermediate species. Examples of strong couplings between environment and electronic structure are found in proteins associated with electron [2, 6, 7], proton and hydride transfer [8-10], and noncatalytic ligand binding [3-5]. The trapping of intermediates by a laser field, or the modulation of reaction barriers in molecules dressed in the field, can also be considered particular consequences of using strong external couplings [11, 12].

Our basic notion in this work is that local electric fields might not only modify the effective energy of a molecule, but also affect markedly the electronic properties along the reaction paths. There are a number of nonadiabatic approaches in the literature to study the strong interrelation between molecular structure and electronics [13]. Most approaches aim at describing these processes in such a way that the external agents make possible the transition from reactant to product. Some recent developments employ: (i) classical trajectories that propagate with a probability to "jump" between two relevant (adiabatic) Born-Oppenheimer (BO) surfaces [9, 14-18], (ii) superpositions of adiabatic wave functions coupled to a model of bath dissipation [19-21], and (iii) schemes based on quasi-diabatic solutions, whose residual nonadiabatic coupling terms appear to be the responsible for the chemical process [22-25].

In this work, we extend a recently introduced method (the so-called "generalized electronic diabatic" (GED) approach [26-31]) to analyze trends in entire families of model potential energy surfaces. Here, a nonisolated system (e.g., a molecule in presence of a radiation field) is described by coherent quantum states that are linear superpositions of orthogonal electronic diabatic functions coupled to an external electromagnetic field [26-29]. The minimum set of diabatic functions needed is dictated by the choice of reaction channels of interest, as well as by symmetry requirements imposed by the external coupling. Ideally, these diabatic basis functions should be represented within a scheme that introduces no residual dependence on the nuclear geometry, e.g., by using a fixed atomic orbital grids; in practice, however, one could resort to approximate diabatization schemes used elsewhere [22-25].

To derive general trends, for the moment we can bypass the diabatic functions, and use instead simple diabatic potential energy models. Recently, we have followed this route to discuss the geometry and topology of 1D- and 2D-potential energy surfaces for symmetric isomerizations (e.g., thermoneutral reactions, interconversions of degenerate species) [30, 31]. Here, we will extend these ideas to nonsymmetric processes. In particular, we model endo/exothermic processes, as well as reactions where reactant and products have at least one distinct vibrational mode. Using simple two-dimensional (2D) harmonic models, we show how the energetics can strongly be affected by a coupling to the exterior environment.

Here, we focus on families of 2D-reaction models; in particular, we deal with the generic isomerization between two closed-shell electronic species. When introducing an external quasi-static electric field, the field parity makes it necessary to include a third open-shell diabatic basis function to "bridge" a pair of closed-shell species (reactant and product). In this minimalistic representation, we find a set of three diabatic electronic basis that can be coupled by the external field into a coherent quantum state; the amplitudes of the quantum state in the diabatic basis functions can be modulated in turn by changes in the external field.

In the next sections, we show how this external agent modifies the reaction path geometry and the number of minimum-energy configurations observed (i.e., configurations where the total energy in the field is locally stationary). Conclusions are derived for a large ensemble of relevant parameters that characterize the diabatic model surfaces and the external field [30,31]. As a result, we provide a classification for entire sets of three-state models in terms of the geometry of the total energy surface 
within that field. The GED approach can thus serve as modeling tool which allows us to locate the families of nonisolated chemical systems (or models) with desired particular geometrical features in their total energy surface.

The work is organized as follows. In the next section, we give a brief overview of the GED approach employed in previous works [26-31]; particular emphasis is made on the key role of the environment and the selected model states. Then, we discuss the three-state models for asymmetrical processes. Two later sections present briefly the main trends in endothermic processes involving reactants and products with both degenerate and nondegenerate normal modes, respectively. We close with a summary of conclusions.

\section{Chemical Processes in the Presence of an External Medium: The GED Approach}

In the present conceptual framework, a local environment provides the external electro-magnetic field that modulates a reaction. As it is the case when studying the effects of laser intensity on chemical properties, we assume this field to be quasi-static (i.e., effectively nonoscillating) over the extent of a small chemical system [11, 12]. This field will produce a coherent quantum state that superimposes at least three diabatic states, i.e., a reactant, a product, and a third open-shell "transition state" required by symmetry for the isomerization of two closed-shell species [28, 29]. In our formulation, each electronic diabatic basis function represents a single, distinct electronic (diabatic) species; each such species is characterized by a single local minimum-energy configuration (i.e., a stationary geometry in the corresponding diabatic potential energy surface as represented in laboratory space). In other words, each diabatic state acts as a single attractor for the positive (nuclear) charge field.

In the GED approach, the external field is assumed to be an agent that accounts for [5-10]:

a. Environmental effects that can modify the geometry of the effective (total) potential energy hypersurface vis-à-vis that of an isolated system described by diabatic potentials. The field should be able to furnish the anharmonicities needed to generate all possible surface topologies, including all possible standard and degenerate critical points [31].

b. Environmental effects that allow reaction path geometries with counter-intuitive electronic characteristics (e.g., a product-like electronic signature for "molecular structures" whose geometries are decidedly not product-like). It should be noted that we view the reaction path as a curve defined in the real space (laboratory frame) of positive charge coordinates. The use of a laboratory frame, rather than a molecular frame, provides a more convenient representation of the diabatic electronic states as invariant entities decoupled from the actual location of the nuclear charges.

In a brief overview, the general notions in the algorithm are as follows $[29,30]$ :

1 . We consider a set of $m$ classical positive testcharges (the "nuclei"), which create a Coulomb potential within the electron gas. Their spatial configuration is denoted by $\boldsymbol{\xi}=\left(\boldsymbol{\xi}_{1}, \ldots, \boldsymbol{\xi}_{m}\right)$, with $\boldsymbol{\xi}_{i} \in \mathfrak{R}^{3}$ the vector position of the $i$-th charge in laboratory (real) space.

2. The classical (massless) test-charges define a quantum/classical model hamiltonian:

$$
\hat{\mathbf{H}}_{\mathrm{e}}(\hat{\mathbf{q}}, \xi)=\hat{K}_{\mathrm{e}}(\hat{\mathbf{q}})+\hat{V}_{\mathrm{ee}}(\hat{\mathbf{q}})+\hat{V}(\hat{\mathbf{q}}, \xi),
$$

where $\hat{K}_{\mathrm{e}}(\hat{\mathbf{q}}), \hat{V}_{\mathrm{ee}}(\hat{\mathbf{q}}), \hat{V}(\hat{\mathbf{q}}, \xi)$ are, respectively, the kinetic and Coulomb potential operators of the electronic subsystem, and the interaction operator for the classical and quantum systems. The latter includes the repulsion within the system of classical test charges [28].

3. We consider a complete set of diabatic functions $\left\{\psi_{j}(\mathbf{q})\right\}$, each of which provides a representation for an electronic diabatic chemical species. Physically, each of these diabatic species represents a stable isolated system, with a potential energy attractor given by [28]:

$$
U_{k}(\xi)=U\left(\xi ;\left[\psi_{k}(\mathbf{q})\right]\right)=\left\langle\psi_{k}(\mathbf{q}) \mid \hat{\mathbf{H}}_{\mathrm{e}}(\hat{\mathbf{q}}, \xi) \psi_{k}(\mathbf{q})\right\rangle .
$$

Each diabatic potential in Eq. (2) is a $\xi$-dependent single-minimum attractor, that is, a potential energy function with at a unique vector $\boldsymbol{\xi}^{(k)}$ in laboratory space so that $\left\|\nabla_{\xi} U_{k}\right\|=0$. In the context of this work, each $\boldsymbol{\xi}^{(k)}$-vector represents a stationary geometry of the hamiltonian in Eq. (1). Only at these geometries, the hamiltonian in Eq. (1) coincides with the one used in the standard $\mathrm{BO}$ model; at a stationary geometry $\boldsymbol{\xi}^{(k)}$, both methods will give equivalent results as long as their electronic configuration spaces are isomorphic, and the explicit computations are performed with the same methodology. At geometries other than $\boldsymbol{\xi}^{(k)}$, however, we construct diabatic potential energy functions as in 
Eq. (2), instead of the standard adiabatic BO solutions (see later).

4. We regards the $\left\{\psi_{j}(\mathbf{q})\right\}$-functions as strictly diabatic, i.e., they maintain their symmetry features for all $\boldsymbol{\xi}$-configurations; in contrast, the $U_{j}(\boldsymbol{\xi})$-potential changes in $\xi$-space as a confining attractor. The $\left\{\psi_{j}(\mathbf{q})\right\}$-set is a basis for building the coherent quantum states that describe the chemical process at issue.

5. The $\left\{\psi_{j}(\mathbf{q})\right\}$-set can be regarded as the eigenfunctions of a particular form of the hamiltonian in Eq. (1), i.e., where the $\boldsymbol{\xi}$-geometry is kept at a $\left\|\boldsymbol{\xi}^{(k)}\right\|$ attractor. Each of these attractors correspond to equilibrium ("stationary") geometries of relevant reactants or products determined by $\left\|\nabla_{\xi} U_{k}\right\|=0$ :

$\hat{\mathbf{H}}_{\mathrm{e}}\left(\hat{\mathbf{q}}, \xi^{(k)}\right) \psi_{j}(\mathbf{q})=E_{j}\left(\xi^{(k)}\right) \psi_{j}(\mathbf{q})$, with $E_{k}\left(\xi^{(k)}\right)=U_{k}\left(\xi^{(k)}\right)$.

In practice, it might be sometimes more convenient to build a $\left\{\psi_{j}(\mathbf{q})\right\}$-set that includes functions computed at different attractors. Still, since the $\left\{\psi_{j}(\mathbf{q})\right\}$-functions are diabatic, they will be common solutions to all single point hamiltonians $\hat{\mathbf{H}}_{\mathrm{e}}\left(\hat{\mathbf{q}}, \xi^{(k)}\right)$, computed at different $\left\|\nabla_{\xi} U_{k}\right\|=0$ attractors. Note however that, although each $\hat{\mathbf{H}}_{\mathrm{e}}\left(\hat{\mathbf{q}}, \xi^{(k)}\right)$ operator can generate the entire set of $\left\{\psi_{j}(\mathbf{q})\right\}$-functions, the corresponding $\left\{\mathrm{E}_{j}\left(\boldsymbol{\xi}^{(k)}\right)\right\}$-eigenvalues will be ordered differently at distinct $\boldsymbol{\xi}^{(k)}$-attractors.

6. The $\left\{U_{k}(\xi)\right\}$-potentials can be modeled, in a first approximation for isomerization reactions, as harmonic (quadratic) forms in $\boldsymbol{\xi}$. This approach is enough to capture all important features in symmetric 2D chemical processes. Recent work indicates that, although each $U_{k}(\xi)$ is harmonic, an external field coupling provides all anharmonicities required to describe the effective energy surface of a nonisolated system [30, 31].

7. The diabatic $\left\{\mid \psi_{j}(\mathbf{q})>\right\}$-states can be coupled with an external agent in various ways depending on their symmetry. When using a quasi-static (electric) radiation field, we can couple closed-shell and open-shell singlet states [29, 30]; different spin states will interact when using the electronic spinorbit $\mathbf{L} \cdot \mathbf{S}$ operator or a nuclear spin coupling [32]. Here, we expand the kinetic-energy operator in powers of the vector potential $\mathbf{A}$; in the limit of weak external fields, the stationary Schrödinger equation for nonisolated systems becomes [26]:

$$
\begin{array}{r}
\hat{H}_{\text {full }} \Phi(\mathbf{q} ; \xi)=E_{\text {full }}(\xi) \Phi(\mathbf{q} ; \xi), \hat{H}_{\text {full }}=\hat{\mathbf{H}}_{\mathrm{e}}(\hat{\mathbf{q}}, \xi)+\hat{V}_{e-\text { field }} ; \\
\hat{V}_{e-\text { field }} \approx-(e / m c) \mathbf{A} \cdot \hat{\mathbf{p}} . \quad \text { (4) }
\end{array}
$$

8. The actual $\mid \Phi>$-states of the nonisolated quantum system can now be represented as coherent superpositions within the diabatic basis $\left\{\mid \psi_{j}(\mathbf{q})>\right\}$ states:

$$
\begin{array}{r}
\Phi(\mathbf{q} ; \xi, \mathrm{A})=\sum_{s} c_{s}(\xi, \mathbf{A}) \psi_{s}(\mathbf{q})=\left[\psi_{1}(\mathbf{q}), \ldots, \psi_{i}(\mathbf{q}) \ldots\right] \\
\quad \times\left[c_{1}(\xi, \mathrm{A}), \ldots, c_{i}(\xi, \mathrm{A}) \ldots\right]^{t},
\end{array}
$$

with $\left[\psi_{1}(\mathbf{q}), \ldots, \psi_{i}(\mathbf{q}) \ldots\right]$ the row vector of the diabatic basis; the $\left\{c_{k}(\boldsymbol{\xi}, \mathbf{A})\right\}$-coefficients describe their coupling in all external fields (i.e., the electromagnetic vector potential $\mathbf{A}$ and the classical test charges in $\boldsymbol{\xi}$-configuration). Within these fields, each $\mid \Phi>$-state produces the following effective potential energy after integrating over electronic qcoordinates:

$$
E_{\text {full }}(\xi, \mathrm{A})=\left\langle\Phi(\mathbf{q} ; \xi, \mathrm{A}) \mid \hat{H}_{\text {full }} \Phi(\mathbf{q} ; \xi, \mathrm{A})\right\rangle_{q} .
$$

Later on, we discuss the geometrical properties of reaction paths on model 2D potential energy surfaces on $(x, y)$-space; for simplicity, the latter will be denoted simply as $E_{\text {full }}(x, y)$.

Within the present conceptual framework, a chemical process can be described as a shift in the $\left\{\left|c_{k}\right|\right\}$-coefficients, which represent the amplitudes of the quantum state $|\Phi(\mathbf{q} ; \boldsymbol{\xi}, \mathbf{A})\rangle$ in the diabatic basis functions. Each squared amplitude can also be related to the response intensity (probability) that a given chemical species, or reaction channel, appear when performing laboratory measurements at the $\xi$-geometry [28]. In this sense, the $\left\{\left|c_{k}\right|^{2}\right\}$-intensities give information on the population of individual (diabatic) electronic species that can be observed with a given set-up.

As one modulates the external field couplings (i.e., the $\left\{V_{i j}\right\}$-matrix elements), or reconfigures the test charges in $\boldsymbol{\xi}$-space, the electronic characteristics of the $\mid \Phi(\mathbf{q} ; \boldsymbol{\xi})>$-state will vary. The transition amplitude $\left\langle\psi_{i}(\mathbf{q}) \mid \hat{\mathbf{p}} \psi_{j}(\mathbf{q})\right\rangle_{q}$ must be different from zero in order to change the general quantum state $|\Phi\rangle$. Thus, if the $i$ - and $j$-states have the same q-parity, this integral is necessarily zero. For this reason, we need a third state, namely, the "transition state." When using a single-determinantal form for the diabatic basis functions, this symmetry constraint implies an open-shell representation for the transition state.

By monitoring the $\left\{\left|c_{k}\right|^{2}\right\}$-intensities along reaction paths, we compare different structures in configurational space in terms of their electronic simi- 
larity, rather than geometrical similarity. In the usual approach, two reaction individual geometries along a path, say $\boldsymbol{\xi}_{1}$ and $\boldsymbol{\xi}_{2}$, can be compared in terms of the Euclidean distance $D_{12}=\left\|\boldsymbol{\xi}_{2}-\boldsymbol{\xi}_{1}\right\|$ or the geodesic arc length over the potential energy surface $E_{\text {full }}(\boldsymbol{\xi}, \mathbf{A})$. In contrast, we can compare now these two geometries by using an electronic similarity "distance" $D_{12}^{(e)}$ defined as follows:

$$
D_{12}^{(e)}=\left[\sum_{s}\left(\left|c_{s}\left(\xi_{2}\right)\right|-\left|c_{s}\left(\xi_{1}\right)\right|\right)^{2}\right]^{1 / 2}
$$

where the $\left\{\left|c_{s}\left(\boldsymbol{\xi}_{j}\right)\right|\right\}$-amplitudes define the quantum state $\left|\Phi\left(\mathbf{q} ; \boldsymbol{\xi}_{j}\right)\right\rangle$ at a $\boldsymbol{\xi}_{j}$-geometry in Eq. (5).

In summary, our approach decouples the notion of electronic similarity from that of geometrical distance along the reaction path because a small $D_{12}=\| \xi_{2}-$ $\xi_{1} \|$ value does not necessarily imply a small $D_{12}^{(e)}$ value in Eq. (7). If we obtain a $\mid \Phi(\mathbf{q} ; \boldsymbol{\xi})>$-state with a large product-like amplitude for a given pair of $(\boldsymbol{\xi}, \mathbf{A})$ values, it may be possible, depending of the particular laboratory set up, to "harvest" such a quantum state as an actual chemical product out of the reacting system. In contrast, in the standard BO model, a reactant-product conversion appears as a (seemingly unaided) continuous "transformation" of the electronic structure as the nuclei are displaced.

In this work, however, decoherence phenomena (e.g., the ability of the quantum molecular state to "collapse" into a single diabatic species during the course of a reaction) have been excluded from the discussion. They could be incorporated though by introducing a transition probability to a given reaction channel when sampling at each $\boldsymbol{\xi}$-configuration [28].

\section{Two-Dimensional GED Models Built From Three Diabatic States}

We focus on general trends in the geometry and topology for a large class of energy surfaces $E_{\text {full }}(x, y)$ for isomerization processes between closed-shell reactants and products. For the sake of discussion, we assume that most relevant features for one such process (e.g., a keto-enol isomerization $[26,27])$ can be captured in a 2D laboratory space with configurations $\{\boldsymbol{\xi}=(x, y)\}$. The $(x, y)$-frame can be regarded as a laboratory plane which provides a clear distinction between the equilibrium geometries for the three diabatic electronic species; the $(x, y)$-coordinates could represent the position a sin- gle nucleus being transferred along the isomerization reaction path.

As discussed earlier, the simplest description of processes requires three diabatic states: reactant $(R)$, product $(\mathrm{P})$, and an open-shell "transition state" (TS) [29-31]. The latter diabatic |TS $>$-state is associated with an actual diabatic electronic attractor, and it will be coupled with the diabatic $|\mathrm{R}\rangle$ - and $\mid \mathrm{P}>$-states via the external $V_{e \text {-field }}$ operator. Within this minimal approach, quantum states are linear superpositions of three diabatic $\left\{\psi_{i}(\mathbf{q})\right\}$-states, each of which can be thought out as a single Slater determinant. This makes a clear contrast with the standard BO approach, which would involve a single determinant that changes continuously from a reactant-like to a product-like closed-shell function (although such a physical transformation would not be allowed by parity). In this context, intermediate "transition structures" may appear as familiar saddle points on the energy surface. In the GED context, a barrier can also be found, but rather as a geometric feature on the $E_{\text {full }}(x, y)$ surface derived from three diabatic states coupled by the external field.

The present models are constructed by coupling three harmonic diabatic potential energy functions $\left\{U_{i}(x, y)\right\}$ with the external potential. These threestate models are built as follows:

i. Each basis state contributes a single 2D harmonic attractor $U_{i}(x, y)$; the labels $i=1,2,3$ stand for the electronic $|\mathrm{R}\rangle,|\mathrm{P}\rangle$, and $|\mathrm{TS}\rangle$ diabatic species, respectively. As discussed before, each $U_{i}(x, y)$-attractor is characterized by an equilibrium geometry $\boldsymbol{\xi}^{(i)}=\left(x_{i}, y_{i}\right)$.

ii. We are interested in asymmetric isomerizations involving distinct diabatic potential wells $U_{1}$ and $U_{2}$, for reactant and products. These asymmetries may arise from considering endothermic process $\left(\min U_{2}>\min U_{1}\right.$ ), diabatic reactant and products with different force constants, or a diabatic $\mid \mathrm{TS}>$-state with a stationary geometry asymmetrically placed with respect to the reactant and product attractors. We consider the following forms for the diabatic harmonic potentials (with dimensionless parameters):

$$
\begin{gathered}
U_{1}(x, y)=\frac{1}{2} k_{x}^{(1)} x^{2}+\frac{1}{2} k_{y}^{(1)} y^{2}, \\
U_{2}(x, y)=\Delta_{2}+\frac{1}{2} k_{x}^{(2)}(x-3)^{2}+\frac{1}{2} k_{y}^{(2)}(y-3)^{2}, \\
\Delta_{2} \geq 0,
\end{gathered}
$$




$$
\begin{array}{r}
U_{3}(x, y)=\Delta_{3}+\frac{1}{2} k_{x}^{(3)}\left(x-x_{3}\right)^{2}+\frac{1}{2} k_{y}^{(3)}\left(y-y_{3}\right)^{2}, \\
\Delta_{3} \geq 0,
\end{array}
$$

where the electronic diabatic reactant is given the conventional equilibrium geometry of $\boldsymbol{\xi}^{(1)}=(0,0)$ whereas the stationary geometry for the electronic diabatic product is $\boldsymbol{\xi}^{(2)}=(3,3)$.

iii. Below, we consider cases with a symmetrically located $U_{3}$ (i.e., $\left.\boldsymbol{\xi}^{(3)}=(3 / 2,3 / 2)\right)$, as well as a representative asymmetric placement $\left(\boldsymbol{\xi}^{(3)}=(5 / 2\right.$, $-1)$ ). We consider also other asymmetric processes: (a) a series of endothermic transformations (i.e., where $\Delta_{2}>0$ increases) between a pair of reactant and product with degenerate normal modes (with $\left.k_{x}^{(1)}=k_{y}^{(1)}=k_{x}^{(2)}=k_{y}^{(2)}=8\right)$, and (b) a thermoneutral transformation $\left(\Delta_{2}=0\right)$ between two distinct reactant and products (here, $k_{x}^{(1)}=k_{x}^{(2)}=k_{y}^{(2)}=8$ and $k_{y}^{(1)}$ $=32$ ). In the latter case, we use the asymmetricallyplaced TS-state (with $\left(\boldsymbol{\xi}^{(3)}=(5 / 2,-1)\right)$. In all processes we have considered a high-energy diabatic TS-attractor with $\Delta_{3}=23$ and $k_{x}^{(3)}=k_{y}^{(3)}=15$. These parameters are sufficient to extract illustrative trends that will be valid over for a large family of diabatic model potentials.

iv. The general quantum states for the nonisolated three-state model system become:

$\Phi(\mathbf{q} ; \xi, \mathrm{A})=c_{1}(\xi) \psi_{1}(\mathbf{q})+c_{2}(\xi) \psi_{2}(\mathbf{q})+c_{3}(\xi) \psi_{3}(\mathbf{q})$.

Parity considerations ensure that closed-shell diabatic reactant and products will not couple in the external field, $V_{12}=\left\langle\psi_{1} \mid \hat{V}_{e-\text { field }} \psi_{2}\right\rangle=0$ [26-29]; moreover, the diabatic functions will be orthogonal. By choosing an open-shell $\mid \psi_{3}>$-state, we have nonzero $V_{13}$ and $V_{23}$ couplings in the field; strict diabaticity also ensures that these matrix elements are also $\boldsymbol{\xi}$-independent.

$\mathrm{v}$. The hamiltonian matrix for the effective potential energy in the field becomes then:

$$
\left(\left[\hat{H}_{\text {full }}\right]_{i j}\right)=\left(\begin{array}{ccc}
U_{1} & 0 & V_{13} \\
0 & U_{2} & V_{23} \\
V_{13} & V_{23} & U_{3}
\end{array}\right) ;
$$

the $E_{\text {full }}(x, y)$ surface is built by finding the lowest eigenvalue matrix of $\left(\left(\left[\hat{H}_{\text {full }}\right]_{i j}\right)\right)$ for each $(x, y)$-point. Finally, the amplitudes for the general quantum states are derived as:

$$
\left|c_{1}\right|=\left|V_{13} c_{3}\right| /\left|E_{\text {full }}-U_{1}\right|,
$$

$$
\begin{gathered}
\left|c_{2}\right|=\left|V_{23} c_{3}\right| /\left|E_{\text {full }}-U_{2}\right| \\
\left|c_{3}\right|=\left\{\left(V_{13} /\left|E_{\text {full }}-U_{1}\right|\right)^{2}+\left(V_{23} /\left|E_{\text {full }}-U_{2}\right|\right)^{2}\right. \\
+1\}^{-1 / 2} .
\end{gathered}
$$

As commented before, a $|\mathrm{R}>\rightarrow| \mathrm{P}>$ transition will now be recognized by a sharp change in $\left\{\left|c_{i}\right|\right\}$-coefficients along the reaction paths. Such transition will not only depend on the energetics of the individual diabatic states, but also on the $\left\{V_{i j}\right\}$-couplings. For simplicity, we consider nonzero symmetric couplings (i.e., $V_{13}=V_{23}$ ). By systematically varying $V_{i j}$ and the placement of the $U_{3}$-minimum, we can monitor the properties of $E_{\text {full }}(x, y)$-surfaces that arise within a large ensemble of possible models. To this end, we classify $E_{\text {full }}(x, y)$-surfaces by using topological features (e.g., the number and types of nondegenerate critical points [30]), and then we study which surfaces share these characteristics on a convenient parameter space for models. For simplicity, we choose two parameters: (a) the $V_{13}=V_{23}$ value, and (b) the distance $d_{3}=\left[\left(x_{\text {mid }}-x_{3}\right)^{2}+\left(y_{\text {mid }}-y_{3}\right)^{2}\right]^{1 / 2}$, where $\boldsymbol{\xi}_{\text {mid }}=$ $\left(x_{\text {mid }}, y_{\text {mid }}\right)$ is the midpoint of the line joining the minima of $U_{1}$ and $U_{2}$. With the choices of minima made here, $d_{3}$ will measure the distance to the $x=y$ line that joins the latter minima. We shall consider the $\left(d_{3}, V_{i 3}\right)$-space a "phase diagram," in the sense that all $E_{\text {full }}(x, y)$-models that share the same topological features over a range of $\left(d_{3}, V_{i 3}\right)$-values will belong to the same class (or "phase") of model reactions. By knowing the type, number, and location of these "phases," it would be possible to simulate a desired reaction process.

\section{Properties of Reaction Paths in Symmetric Models of Endothermic Processes}

We consider first the case of symmetric transformations, where $k_{x}^{(1)}=k_{y}^{(1)}=k_{x}^{(2)}=k_{y}^{(2)}=8$, and $k_{x}^{(3)}$ $=k_{y}^{(3)}=15$. (These parameters characterize a particular set of diabatic states.)

Symmetry is also ensured by placing the $\min U_{3}$ attractor along the line connecting the minima for the diabatic reactant and product, i.e., $y=x$, and equidistant to both. In the case $d_{3}=0$, the $\mid$ TS $>$ state minimum is midway at $\boldsymbol{\xi}^{(3)}=(3 / 2,3 / 2)=$ $\xi_{\text {mid }} ; d_{3}>0$ values correspond to a $\mid \mathrm{TS}>-$ minimum displaced along $y=3-x$ with $x>3 / 2$ (i.e., along the perpendicular bisector to the line joining the minima of $U_{1}$ and $U_{2}$ ). Endothermicity is intro- 
duced by increasing the $\Delta_{2}$ value. For each set of parameters $d_{3}$, we have mapped the $E_{\text {full-surfaces }}$ over the $(x, y)$-plane in laboratory space. In all cases, we have computed all nonsingular critical points (minima, maxima, and saddle points), as well as the minimum-energy paths connecting pairs of minima by a type-1 saddle point. In particular, we locate the Fukui path, i.e., the shortest geodesic on $E_{\text {full }}(x, y)$ between reactant and product minima. For the sake of classification, the numbers of nondegenerate critical points are labeled as $N_{\mathrm{m}}, N_{\mathrm{s}}$, and $N_{\mathrm{M}}$, for minima, saddle points, and maxima, respectively. Another important piece of information is $N_{\mathrm{s}}^{(F)}$, the number of type-1 saddle points traversed by the Fukui path, i.e., the minimum energy reaction path joining the reactant-like and product-like minimum. Note that the latter are minima of the effective energy surface in the field, $E_{\text {full }}(x, y)$; they may not coincide in general with the diabatic $\boldsymbol{\xi}^{(1)}$ and $\boldsymbol{\xi}^{(2)}$ minima, and they may even disappear for sufficiently large values of the external field.

Figure 1 shows representative 2D contours for increasingly endothermic model effective $E_{\text {full }}(x, y)$ surfaces. The four diagrams in Figure 1 correspond to models with the same $d_{3}=0$ and $V_{13}=V_{23}=9$ parameters (in dimensionless units), yet different $\min U_{2}=\Delta_{2}$ values. The location of the diabatic stationary $\left\{\tilde{\boldsymbol{\xi}}^{(i)}\right\}$-geometries are denoted by the black circles.

The first three diagrams in Figure 1 share the same geometrical characteristics, namely the $E_{\text {full }}{ }^{-}$ surfaces have three local minima, connected by a single (minimum-energy) Fukui path, $N_{\mathrm{s}}=N_{\mathrm{s}}^{(F)}=$ 1. The open (white) circles indicate the location of the local minima in the field, whereas the white square shows the location of the type- 1 saddle point; the Fukui path is indicated by the thick straight line joining the $\mid R>$-like and $\mid \mathrm{P}>$-like minima. In contrast, the last diagram, corresponding to the more endothermic process $\left(\Delta_{2}=25\right)$, has an $N_{\mathrm{s}}$ $=0$ topology and no Fukui path. In this case, the diabatic product is located above the diabatic $\mid$ TS $>$ state with $\Delta_{3}=23$, and thus it is less accessible to reaction paths.

In all cases, we have classified all points on $(x, y)$ space in terms of which diabatic state has the largest $\left|c_{i}\right|^{2}$-intensity. The boundary between these regions are represented by dashed lines. The central region represents the area dominated by the diabatic $\mid \mathrm{TS}>$-state.

The behavior in Figure 1 is representative of some trends found in endothermic processes:
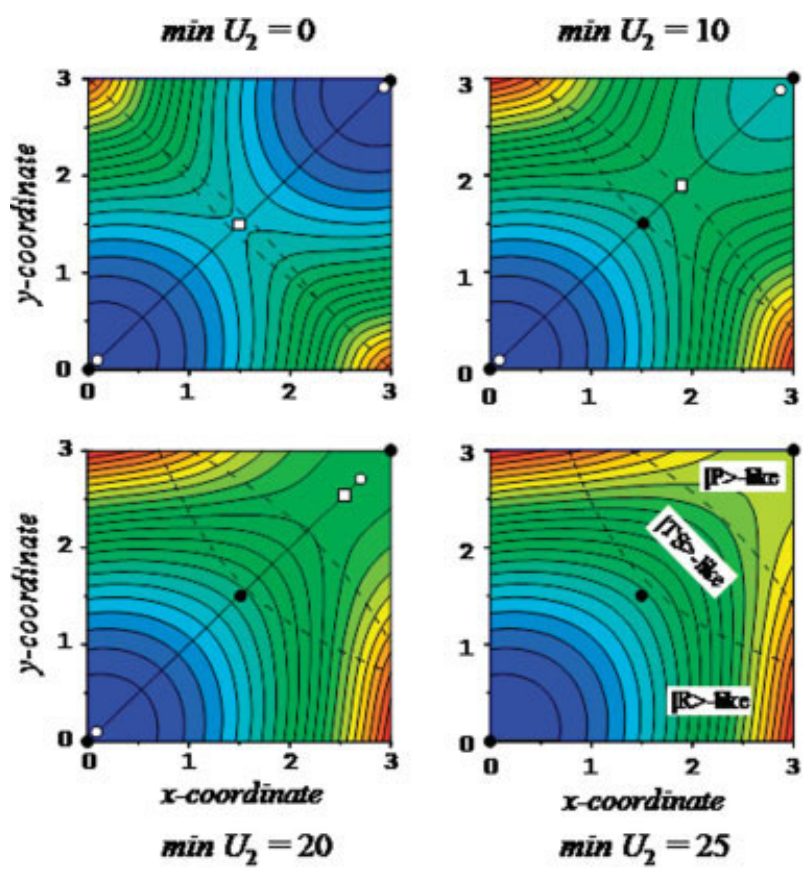

FIGURE 1. Two-dimensional energy contours for a series of symmetric $E_{\text {full }}(x, y)$-models built with three diabatic states coupled in an external field. The diabatic attractors for the reactant and transition state are kept constant in all diagrams, but the diabatic product is raised in energy from $\min U_{2}=\Delta_{2}=0$ up to 25 (see text). The off-diagonal couplings are also kept constant at $V_{13}=V_{23}=9$. The black circles give the location of the diabatic minima, while the white circles give the local minima in the $E_{\text {full }}{ }^{-}$ surface dressed in the field. The white square gives the location of the type-1 saddle point along the Fukui reaction path (indicated with a thick straight line). The dashed lines divide configurational $(x, y)$-space into regions where a single diabatic $\left|c_{j}\right|^{2}$-intensities dominates; the $\mid T S>$ dominated region is in the middle of the diagram. The latter becomes larger with the field intensity, though the geometrical saddle point is shifted towards the $\mid \mathrm{P}>$-dominated region. To avoid cluttering, the regions dominated by a given diabatic basis state are labeled only on the last diagram; the relative position of these regions is the same on all contour maps. The pattern of critical point shift (as a function of the $V_{13}$ ) agrees with the Hammond postulate. See text. [Color figure can be viewed in the online issue, which is available at www.interscience.wiley.com.]

1. For a given coupling, the Fukui path shrinks as the reaction becomes more endothermic; the effective $\mid \mathrm{P}>$-like minimum moves away from the diabatic $\mid \mathrm{P}>$-like minimum.

2. Increasingly more endothermic reactions exhibit a shift in the location of the saddle point of the effective $E_{\text {full }}$-surface, whereby the former ap- 
proaches the $\mid \mathrm{P}>$-like minimum. This behavior is in agreement with the well-known Hammond postulate $[29,33,34]$.

3. The Fukui reaction path can traverse regions of configuration $(x, y)$-space which are dominated by the squared amplitude of the diabatic $\mid$ TS $>$-state, i.e., $\left|c_{3}\right|^{2}>\left|c_{i}\right|^{2}$, with $i=1,2$; these appear as central regions enclosed in dashed lines. In thermoneutral processes $\left(\Delta_{2}=\min U_{2}=0\right)$, this region is small and encloses the effective saddle point. In endothermic models, however, the $\mid \mathrm{TS}>$-dominated region becomes increasingly larger, although eventually it excludes the saddle point. In other words, the "transition structure" geometry will resemble electronically the product, even though Fukui reaction path contains a large section of configurations which resemble electronically the diabatic transition state.

4. Eventually, we find no longer a saddle point when $\Delta_{2}>\Delta_{3}$, although still there is a large section of $(x, y)$-space that is dominated by the electronic signature of the diabatic |TS $>$-state. As well, there is a large region dominated by the electronic structure of the diabatic product, even though there is no longer a $\mid \mathrm{P}>$-like minimum in the effective $E_{\text {full }}$-surface.

These observations characterize a large class of model surfaces. However, as one changes the coupling with the field and the location of the diabatic |TS $>$-attractor, other topologies become possible. Figure 2 illustrates this idea by showing the phase diagram of three-state models with constant endothermicity $\left(\Delta_{2}=10\right)$, yet different $V_{i 3}$ and $d_{3}$ values. The various regions represent different topologies of the $E_{\text {full-surface, classified in terms of the saddle point }}$ numbers $N_{\mathrm{s}}$ and $N_{\mathrm{s}}^{(F)}$. The gray areas correspond to those "phases" (or phase sections) for $E_{\text {full }}$ models whose reaction paths visit configurations dominated by the amplitude in the diabatic |TS $>$-state. This phase diagram illustrates the following trends:

1. Sufficiently strong couplings produce a single-minimum effective surface $\left(N_{\mathrm{s}}=0\right)$. This situation represents a molecular system trapped in the external field; in this case there are no Fukui paths, but the relaxation of this minimum will be dominated by the $\left|c_{3}\right|^{2}$-squared amplitude.

2. At low couplings and nearly symmetric models (i.e., small $d_{3}$ values), we find the simpler surfaces with $\mid \mathrm{R}>$ - and $\mid \mathrm{P}>$-like minima connected by a Fukui path that traverses a single saddle point (i.e., $N_{\mathrm{s}}=1$, $\left.N_{\mathrm{m}}=2, N_{\mathrm{s}}^{(F)}=1\right)$. At higher $\left(d_{3}, V_{i 3}\right)$-values, we find another $N_{\mathrm{s}}=1, N_{\mathrm{m}}=2$ region; however, this "phase" exhibits no reaction path connecting $\mid \mathrm{R}>$ and $|\mathrm{P}\rangle$, but only paths leading from the $\mid R>$-like minimum to the

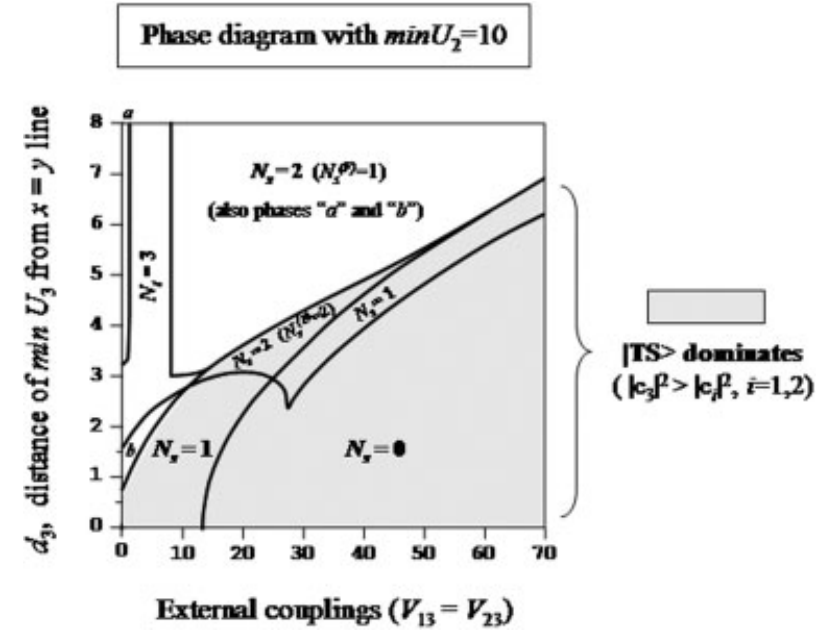

FIGURE 2. "Phase diagram" for symmetric endothermic model $E_{\text {full }}(x, y)$-surfaces with $\Delta_{2}=10$. The 2D parameter space is defined by the external couplings $\left(V_{13}\right.$ $=V_{23}$ ), and $d_{3}$ (the deviation of the diabatic $\mid$ TS $>-$ minimum from the midpoint $\xi_{\text {mid }}=\left(x_{\text {mid }}, y_{\text {mid }}\right)=(3 / 2,3 / 2)$ along the $y=3-x$ line). The $\xi_{\text {mid }}$ point coincides with the $U_{1}=U_{2}$ crossing in a thermoneutral reaction. Phases are labeled with the number of saddle points $\left(N_{\mathrm{s}}\right)$ in $E_{\text {full }}(x, y)$, and the number of saddle points traversed in the Fukui path $\left(N_{s}^{(F)}\right)$. The gray areas indicate "phases" with Fukui paths that have a nonzero section dominated by the $\left|c_{3}\right|^{2}$ squared amplitude. See text for a discussion about the meaning of the different phases and their interconversion.

|TS >-like intermediate (i.e., $N_{s}^{(F)}=0$, because there is no Fukui path).

3. Surfaces with fully connected three minima (i.e., $N_{\mathrm{s}}=3, N_{s}^{(F)}=1$ ) can only appear in a narrow region of model parameters. Over a critical value of $V_{\beta} \approx 8$, the direct connection between the $\mid \mathrm{TS}>-$ and $\mid \mathrm{P}>$-like minima is severed (i.e., $N_{\mathrm{s}}=2, N_{\mathrm{s}}^{(F)}=$ 1). A lowering of the external field intensity can also lead to an intermediate situation with Fukui paths connecting the $\mid \mathrm{R}>$ - and $\mid \mathrm{P}>$-like minima via the intermediate $\mid \mathrm{TS}>$-minimum (i.e., $N_{\mathrm{s}}=2, N_{s}^{(F)}=2$ ). As expected, the latter "phase" exhibits sections of reaction paths dominated by the diabatic |TS $>$-amplitude (gray area), whereas this is not found in the $N_{\mathrm{s}}=2, N_{s}^{(F)}=1$ "phase."

Figure 3 illustrates how the phase diagrams simplify as the reaction models become increasingly more endothermic. In Figure 3, we show the case of a reaction with a diabatic $\mid \mathrm{P}>$-state whose attractor has a minimum energy over that of the minimum for the $\mid \mathrm{TS}>$-attractor. In these models, the possibility to find three local minima fully intercon- 


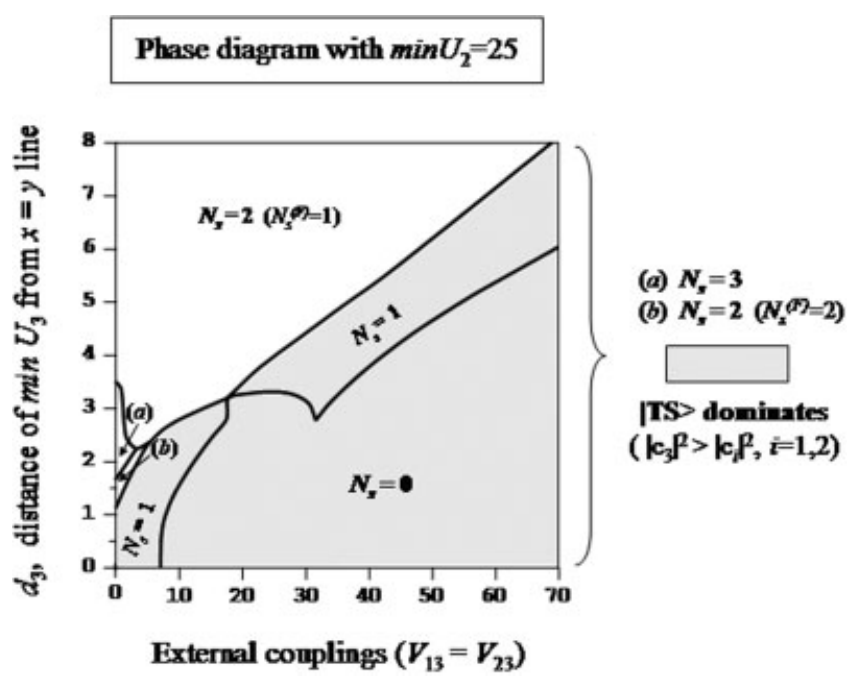

FIGURE 3. Phase diagram for symmetric endothermic $E_{\text {full }}(x, y)$-surfaces with $\Delta_{2}=25$. This $\Delta_{2}$ value puts the minimum of the diabatic $\mid \mathrm{P}>$-attractor above that of the diabatic |TS $>$-state. For this reason, the topology of the effective energy surface simplifies extensively as the external coupling increases (cf. Fig. 2). For symbols, labels, and shading, see Figure 2.

nected by reaction paths is very limited [region (a)], so is the possibility of reaching a $\mid \mathrm{P}>$-like minimum via a $\mid$ TS $>$-like minimum [region (b)]. In the latter case, the allowed $V_{i 3}$ couplings leading to this "phase" are so small that the reaction paths are never dominated by the diabatic $\mid \mathrm{TS}>$-state (i.e., it is outside the area shaded in gray). An increase of the coupling brings a collapse of two saddle points and the $\mid$ TS $>$-minimum, thus producing the smaller $N_{\mathrm{s}}=1$ region in Figure 3. As the figure suggests, some $E_{\text {full }}$-topological "phases" may altogether disappear in very endothermic reactions.

\section{Effective Endothermic Processes in Asymmetric Diabatic Models}

The examples in the previous section correspond to model reactions built with "endothermic" diabatic $U_{1}$ and $U_{2}$-attractors; endothermicity is maintained after coupling them in the external field. It is possible also to produce effective $E_{\text {full }}$-surfaces that are endothermic within the field, even when the diabatic $(|\mathrm{R}>,| \mathrm{P}>)$-pair consists of thermally neutral attractors. To this end, it is sufficient to introduce an asymmetrically placed |TS $>$-attractor, even if the diabatic couplings with the external fields are symmetrical (i.e., still $V_{13}=V_{23}$ ).
Figure 4 illustrates this notion with a series of representative potential-energy contours for 2D asymmetric models. In this case, we find two asymmetries: (a) The $\mid \mathrm{TS}>$-attractor has a minimum placed at $\boldsymbol{\xi}^{(3)}=(5 / 2,-1)$, other parameters being the same as in "Properties of Reaction Paths in Symmetric Models of Endothermic Processes" Section; (b) The diabatic $\mid \mathrm{R}>$ - and $\mid \mathrm{P}>$-attractors are thermally neutral, but the reactant has distinct normal mode frequencies (here, $k_{x}^{(1)}=8, k_{y}^{(1)}=32$ ). This asymmetry yields a diabatic crossing $U_{1}=U_{2}$ at $\xi_{\mathrm{dc}}$ $=\left(x_{\mathrm{dc}}, y_{\mathrm{dc}}\right)=(1.81868,0.83375)$; this point is indicated by a star in Figure 4.

The diagrams in Figure 4 correspond to potential energy surfaces with increasing external field intensity, and they are representative of general trends observed in 3-state models with asymmetric normal modes and an asymmetrically placed diabatic |TS>-attractor:

1. At low external couplings, the $|\mathrm{R}>\rightarrow| \mathrm{P}>$ reaction path begins along the softest normal mode (here, along the $y \approx y_{1}$ direction). This is the case of
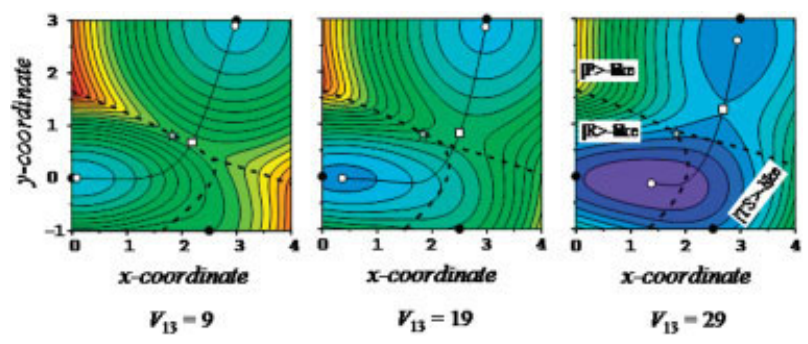

FIGURE 4. Two-dimensional energy contours for a series of asymmetric $E_{\text {full }}(x, y)$-models built with three diabatic states coupled in an external field. The diabatic $\mid R>$-attractor has two distinct normal modes, while the diabatic $\mid \mathrm{P}>$-attractor is symmetric; otherwise, these two potentials are thermally neutral. The diabatic $\mid$ TS $>$ attractor is high in energy, and its minimum is asymmetrically places at $\xi^{(3)}=(5 / 2,-1)$. All other symbols for critical points, reaction paths and electronic-similarity partitioning of $(x, y)$-space are as in Figure 1. To avoid cluttering, the regions dominated by a given diabatic basis state are labeled only on the last diagram; the relative position of the three regions is the same in the three contour maps. Note that, as the external field increases with $V_{13}=V_{23}$, the reaction becomes endothermic, the reaction path changes its curvature, the critical point is shifted, and the |TS $>$-like region becomes larger. Eventually, the Fukui path visits the |TS >-dominated region even when the saddle point (white square) enters the $\mid \mathrm{P}>$-dominated region. [Color figure can be viewed in the online issue, which is available at www.interscience.wiley.com.] 
the weakest coupling, $V_{13}=V_{23}=9$, on the lefthand side of Figure 4 . If the diabatic $U_{3}$-function is asymmetrically placed as in Figure 4, a stronger coupling to the external field curves the path towards the $\mid \mathrm{TS}>$-attractor.

2. Given that the minimum of the diabatic $\mid$ TS $>$ attractor is closer to that of the $\mid \mathrm{R}>$-attractor, increasing external couplings render the effective potential energy endothermic. Along with this change in energetics, we observe that the type- 1 saddle point (white square) shifts towards the $\mid \mathrm{P}>$-like minimum, as expected within validity of the Hammond postulate.

3. As seen in "Properties of Reaction Paths in Symmetric Models of Endothermic Processes" section, the external field shrinks the reaction path, thus making the $\mid \mathrm{P}>$-like minimum approach the saddle point along the Fukui path. Moreover, the $\mid \mathrm{R}>$-like minimum shifts closer to the location of the $\mid \mathrm{TS}>$-attractor. A higher $V_{13}$ value would thus produce eventually a direct path between a $\mid \mathrm{TS}>$ like minimum and a $\mid \mathrm{P}>$-like minimum. Further increase in $V_{13}$ would collapse these critical points and lead to a surface with $\left(N_{\mathrm{s}}=0, N_{\mathrm{m}}=1\right)$ topology. In the present case, the latter topology is observed for $V_{13}>39$.

4. The diabatic $\boldsymbol{\xi}^{(3)}$-minimum is located within the region of configuration $(x, y)$-space dominated by the amplitudes in the diabatic $\mid \mathrm{TS}>$-function (cf. regions limited by dashed lines). At low fields, the Fukui path avoids the latter region, and the saddle point is near the diabatic crossing between reactant and product. As the intensity of the field increases, the reaction path enters the $\mid \mathrm{TS}>$-dominated region, even as the saddle point moves well into the $\mid \mathrm{P}>$-dominated section of configurational space. This interplay between changes in $E_{\text {full }}(x, y)$ geometry and changes in electronic amplitudes mimics the trends seen in "Properties of Reaction Paths in Symmetric Models of Endothermic Processes" section.

Figure 5 highlights these observations in Figure 4 and compares the shifts in Fukui paths over the entire range of external couplings. It is evident that the external field changes the curvature of the reaction path, as well as the placement of the critical points. In particular, the saddle point moves rapidly away from the diabatic crossing (gray star), and the $\mid \mathrm{R}>$-like minimum shifts dramatically along the "soft" normal mode of the reaction. This behavior should be prototypical of 2D simple models with asymmetric modes (e.g., the addition $\mathrm{H}_{2}+\mathrm{CO}$ [27] studied with only two distance coordinates).

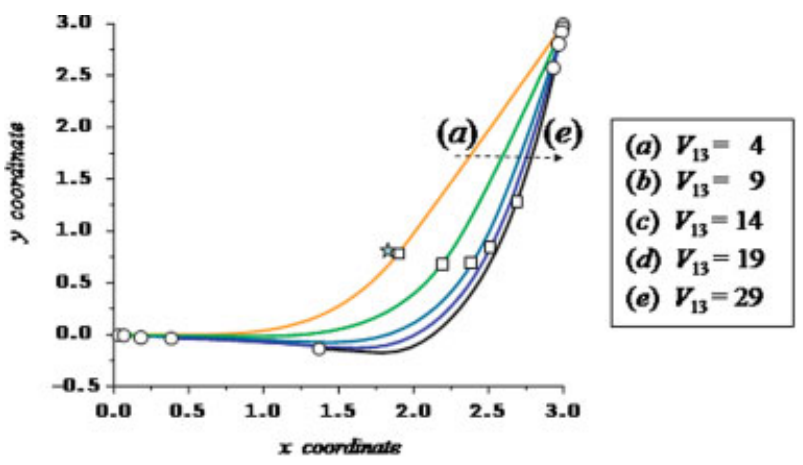

FIGURE 5. Change in reaction path geometry for the asymmetric potential energy surfaces in Figure 4 as a function of the external field. The star in gray indicates the location of the diabatic crossing $\xi_{\mathrm{dc}}=\left(x_{\mathrm{dc}}, y_{\mathrm{dc}}\right)$ between the reactant and product basis states. As the field increases, the reaction path is shifted toward the diabatic $\mid T S>$-state (i.e., the right-hand side of the diagram). The location of the minima for the effective $E_{\text {full }}(x, y)$ surface in presence of the external field are indicated by the open circles, whereas the saddle point along the path is indicated with an open square. Note that the $|R>| P>$ path curves away from zero slope as it leaves the reactant. The curves correspond to external couplings that increase from right to left, from $V_{13}=4$ [first curve, (a)] to $V_{13}=29$ [last curve, (b)]. The intermediate values are listed to the right of the diagram. Note that: (i) the path contracts as the external field becomes larger, (ii) the saddle point and the local $\mid P>$-like minimum approach each other under the same conditions. For $V_{13}>39$, the reaction path disappear and we find a single minimum $\left(N_{\mathrm{s}}=0\right)$. All quantities are dimensionless. [Color figure can be viewed in the online issue, which is available at www.interscience.wiley. com.]

\section{Further Comments and Conclusions}

The contour diagrams in Figures 1 and 4, and the phase diagrams in Figures 2 and 3, are representative of the surface topologies that can be generated by all possible three-state models for endothermic processes (or exothermic ones by permutation). Even though we have employed harmonic diabatic potential, a coupling to an external field appears to be sufficient to generate the geometrical and topological characteristics found in realistic $2 \mathrm{D}$ models with nonperiodic coordinates [35]. It is useful to compare this outcome with that of a standard BO model for a similar process: in this case, there would be a single adiabatic surface that changes from a closed-shell $\mid \mathrm{R}>$-like to a $\mid \mathrm{P}>$-like electronic structure by moving along the reaction path. In the 
GED approach, each geometry along the reaction path is associated with a coherent quantum state which is a linear superposition of three (fixed) diabatic states coupled in an external field. Depending on the intensity of the latter, the amplitudes on each diabatic state will change along the reaction path, thereby opening the possibility of a product reaction channel whenever $\left|c_{2}\right|^{2} \gg\left|c_{i}\right|^{2}$, with $i=1,3$.

As shown here, the geometrical distance of two configurations along a reaction path, $D_{12}$, and the electronic similarity, $D_{12}^{(e)}$ [cf. Eq. (7)], are two distinct notions. Figures 1 and 4 show that two local stationary geometries can be near each other geometrically, yet quite different in electronic signature. The GED approach makes it possible therefore to explain the occurrence of "off-path" species, as well as anti-Hammond behavior [29, 33, 34, 36-38], as a limit behavior for particular values of model parameters and external couplings.

By finding trends in the geometry of $E_{\text {full }}$-surfaces over the space of relevant model parameters, our approach retains its usefulness for simulating realistic $2 \mathrm{D}$ isomerization processes, given that the latter should be included within the set of all possible models. Moreover, we have shown recently that harmonic models can capture even subtle effects associated with the field observed in actual ab initio diabatic functions [31]. Our analysis provides therefore a tool to select those excited states that can play a key role as "transition states" for a given reaction channel, as well as those required to explain the spectroscopic responses of reactant species. Finally, our protocol also allows us to estimate the extent to which a surrounding media modulates the outcome of the reaction.

\section{ACKNOWLEDGMENTS}

G.A.A. acknowledges the Canada Research Chairs' Program, as well as the hospitality of the Department of Physical Chemistry (Uppsala).

\section{References}

1. Fersht, A. Structure and Mechanism in Protein Science; Freeman: New York, 1998.

2. Daniel, R. M.; Dunn, R. V.; Finney, J. L.; Smith, J. C. Annu Rev Biophys Biomol Struct 2003, 32, 69.

3. Ackers, G. K. Adv Prot Chem 1998, 51, 185.

4. Hogle, J. M. Annu Rev Microbiol 2002, 6, 677.
5. Whitten, S. T.; García-Moreno, B. E.; Hilser, V. J. Proc Natl Acad Sci USA 2005, 102, 4282.

6. Winkler, J. R.; Wittung-Stafshede, P.; Leckner, J.; Malmström, B. G.; Gray, H. B. Proc Natl Acad Sci USA 1997, 94, 4246.

7. Pascher, T.; Chesick, J. P.; Winkler, J. R.; Gray, H. B. Science 1996, 271, 1558.

8. Meuwly, M.; Karplus, M. Biophys J 2004, 86, 1987.

9. Watney, J. B.; Soudackov, A. V.; Wong, K. F.; HammesSchiffer, S. Chem Phys Lett 2006, 418, 268.

10. Petrov, E. G.; Teslenko, V. I.; May, V. Phys Rev E 2003, 68, 061916.

11. Bandrauk, A. D.; Sedik, E.-W. S.; Matta, C. F. J Chem Phys 2004, 121, 7764.

12. Bandrauk, A. D.; Sedik, E.-W. S.; Matta, C. F. Mol Phys 2006, 104, 95.

13. Butler, L. J. Annu Rev Phys Chem 1998, 49, 125.

14. Borgis, D.; Hynes, J. T. Chem Phys 1993, 170, 315.

15. Tully, J. C. In Classical and Quantum Dynamics in Condensed Phase Simulations; Berne, B. J., Ciccotti, G., Coker, D. F., Eds.; World Scientific: Singapore, 1998; p 489.

16. Wong, K. F.; Rossky, P. J. J Phys Chem 2001, 105, 2546.

17. Benkovic, S. J.; Hammes-Schriffer, S. Science 2003, 301, 1196.

18. Käb, G. J Phys Chem 2006, 110, 3197.

19. Hanna, G.; Kapral, R. J Chem Phys 2005, 122, 244505.

20. Kim, H.; Kapral, R. Chem Phys Lett 2006, 423, 76.

21. Kapral, R. Annu Rev Phys Chem 2006, 57, 129.

22. Matsunaga, N.; Yarkony, D. R. Mol Phys 1998, 93, 79.

23. Nakamura, H.; Truhlar, D. J Chem Phys 2001, 115, 10353.

24. Kryachko, E. S. Adv Quantum Chem 2003, 44, 119.

25. Köppel, H. In Conical Intersections: Electronic Structure, Dynamics, and Spectroscopy, Domcke, W., Yarkony, D. R., Köppel, H., Eds.; World Scientific: Singapore, 2004; p 17.

26. Tapia, O. In Quantum Systems in Chemistry and Physics, Vol 2: Advanced Problems and Complex Systems, Hernández-Laguna, A., Maruani, J., McWeeny, R., Wilson, S., Eds.; Kluwer: Dordrecht, 2000; p 193.

27. Tapia, O.; Braña, P. J Mol Struct (Theochem) 2002, 580, 9.

28. (a) Arteca, G. A.; Tapia, O. J Math Chem 2004, 35, 1; (b) Arteca, G. A.; Tapia, O. J Math Chem 2005, 37, 389.

29. Arteca, G. A.; Tapia, O. Int J Quantum Chem 2007, 107, 382.

30. Arteca, G. A.; Rank, J. P.;Tapia, O. Int J Quantum Chem 2008, 108, 651.

31. Arteca, G. A.; Rank, J. P.; Tapia, O. J Theor Comput Chem 2007, 4, 869 .

32. Buchachenko, A. L. J Phys Chem A 2001, 105, 9995.

33. Solà, M.; Toro-Labbé, A. J Phys Chem 1999, 103, 8847.

34. Bulat, F. A.; Toro-Labbé, A. J Phys Chem 2003, 107, 3987.

35. Mezey, P. G. Potential Energy Hypersufaces, Elsevier: Amsterdam, 1987.

36. Matthews, J. M.; Fersht, A. R. Biochemistry 1995, 34, 6805.

37. Lopez, X.; Dejaegare, A.; Karplus, M. J Am Chem Soc 2001, 123, 11755.

38. Wright, C. F.; Lindorff-Larsen, K.; Randles, L. G.; Clarke, J. Nat Struct Biol 2003, 10, 658. 